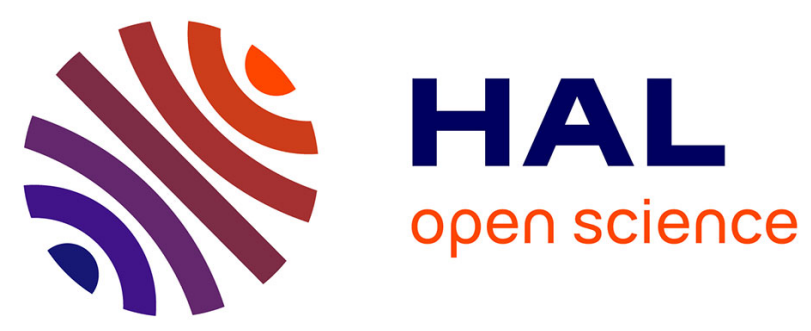

\title{
Blockchain as an Internet of Services Application for an Advanced Manufacturing Environment
}

\author{
Benedito Petroni, Jacqueline Zonichenn Reis, Rodrigo Franco Gonçalves
}

\section{To cite this version:}

Benedito Petroni, Jacqueline Zonichenn Reis, Rodrigo Franco Gonçalves. Blockchain as an Internet of Services Application for an Advanced Manufacturing Environment. IFIP International Conference on Advances in Production Management Systems (APMS), Sep 2019, Austin, TX, United States. pp.389-396, 10.1007/978-3-030-29996-5_45. hal-02460506

\section{HAL Id: hal-02460506 https://hal.inria.fr/hal-02460506}

Submitted on 30 Jan 2020

HAL is a multi-disciplinary open access archive for the deposit and dissemination of scientific research documents, whether they are published or not. The documents may come from teaching and research institutions in France or abroad, or from public or private research centers.
L'archive ouverte pluridisciplinaire HAL, est destinée au dépôt et à la diffusion de documents scientifiques de niveau recherche, publiés ou non, émanant des établissements d'enseignement et de recherche français ou étrangers, des laboratoires publics ou privés. 


\title{
Blockchain as an Internet of Services application for an advanced manufacturing environment
}

\author{
Benedito Cristiano A. Petroni ${ }^{1[0000-0001-9826-1257]}$, Jacqueline Zonichenn Reis ${ }^{10000-0001-}$ \\ 6710-6430] and Rodrigo Franco Gonçalves $2,1[0000-0003-2206-3136]$ \\ ${ }^{1}$ Graduate Studies in Production Engineering, Paulista University, Sao Paulo, Brazil \\ ${ }^{2}$ Politecnic School, University of Sao Paulo, Brazil \\ benedito.petroni@docente.unip.br, zonichenn@hotmail.com,rofranco212@gmail.com
}

\begin{abstract}
In the current dynamic and competitive market, contemporary manufacturing systems must be constantly adapted to meet the requirements for a more agile and smart production. The advent of Industry 4.0 comes as a reference on development of applications and technologies for manufacturing process innovation. Among the pillars of Industry 4.0, a noticeable relevance is given to Cyber Physical Systems, Internet of Things and Internet of Services. In parallel, new technologies as Blockchain and Smart Contracts are important innovations also coined by the Information Technology domain. More specifically, Internet of Services is characterized by a service-oriented computing model enabling a diversity of software-based services through the Internet, among them the Blockchain solution. The paper explores these technologies bringing their intersection as well as their possible applications in the shop floor level. Through the interlock of such concepts, the paper aims to propose an architecture that promotes the utilization of Blockchain for the validation of some service demands in an advanced manufacturing scenario of the Industry 4.0. Lastly a hypothetical case study is presented for illustrating the proposed architecture.
\end{abstract}

Keywords: Blockchain, Internet of Services, Industry 4.0, Cyber Physical Sytems, Smart Contracts

\section{Introduction}

The advent of Industry 4.0 comes as a reference on development of applications and technologies for manufacturing process innovation. Such paradigm integrates Cyber Physical Systems, Internet of Things and Internet of Services [1,2,3].

Individuals and enterprises are each day more surrounded by connected devices and remote services with the potential for real-time interaction. The objects interconnected demand application-centric technologies to be able to provide a diversity of services [4], indicating Internet of Things and Internet of Services domains emerge.

The Internet of Services, for instance, is characterized as a distributed computing environment which can be filled by a large number of software services [5]. 
Blockchain is also considered a new endeavor of the pervasive area of technology information, supporting a secure exchange of data, knowledge and services so that the parties can contribute their parts [6].

By considering that manufacturing have many types of contracts, related with industrial services (e.g. maintenance and support), we can propose the follow question: how could conventional service contracts turn into Smart Contracts?

The aim of this paper is to propose an architecture for the application of Blockchain-technology and Smart Contracts as the linkage between the Internet of Services and the data generation on the shop floor level through the Cyber-Physical Systems.

\section{Research Method}

The paper is theoretical and proposes an architecture of possible implementation, which main goal is the utilization of Blockchain technology for the validation of manufacturing service contracts.

First, through an exploratory bibliography review, the paper brings the technical concepts of Blockchain as well as the rules of use in concern of Smart Contracts. It brings also the state-of-the-art for the technologies subsumed from the Industry 4.0 such as the Cyber Physical Systems, Internet of Things and Internet of Services.

Based on the definitions and funtionalities of these new technologies, an interlock is proposed considering its application on a shop floor scenario. Lastly a hypothetical case study is presented for illustrating the proposed architecture.

\section{$3 \quad$ Blockchain and Smart Contracts}

Blockchain creates reliable transactions through an intelligent code. Such possibility promotes a collaboration in community since the transactions are validated among two or more parties, different from the traditional centralized model [7].

Technically, Blockchain is a protocol or sequence of messages between at least two computers, consisting of algorithms that communicate through the messages. During its operation, it occurs the storage of blocks with information about specific applications, which allow to identify intelligent devices and program them according to the interactions [6].

Blockchain works as a distributed ledger through a system of computers in a network. Its functions are recording transactions, executing electronic contracts and performing asset tracking. There is no central control and no participant is more reliable than any other, depending, therefore, on a decentralized consensus that comes from other participants validation of the existing rules. Such rules, which blockchain can store and validate, are specific domain information called Smart Contracts [8].

Smart Contracts can be understood as a computer protocol used to facilitate and automate financial contracts [9]. According to the operation of the Blockchain, the possibility of storing records with the implementation of Smart Contracts for transactions (application of rules) in distinct domains has become feasible. One of the possible areas of implementation that is setting itself up is the Internet of Services [10]. 


\section{$4 \quad$ Industry 4.0 structural elements}

\subsection{Cyber Physical Systems}

Cyber Physical Systems (CPS) are the convergence of two layers of information technology: the physical or technology layer, and the "cyber" or virtual layer [11].

The architecture of this type of electronic system is composed of sensors and actuators that monitor the physical processes in the environment in which they act, creating a virtual twin of the real world [12]. Sensors are devices responsible for capturing information from the physical environment and transforming it into electrical and digital signals. Actuators perform interventions in the physical world started from digital signals and commands, such as the opening and closing of valves, for example.

The Cyber Physical Systems will be increasingly involved in production systems due to the interconnectivity skills and the autonomy over external influences and internal stored configurations. In such production systems, both horizontal integration through value networks as well as the vertical integration through network manufacturing systems can be built to produce intelligent production [13].

\subsection{Internet of Things}

It is through the Internet of Things that the Cyber Physical Systems communicate with each other and with people in real time [12].

Internet of Things (IoT) is a term created by a British entrepreneur and founder of start-ups called Kevin Ashton. His idea formulated in 1999 described a system in which the material world communicates with computers through the exchange of data with omnipresent sensors. Almost a decade after its creation, in 2009, the number of devices connected to the network exceeded the number of inhabitants of our planet. This time, according to Cisco, is the true birth of the "Internet of Things" [4].

With the advent of IoT, objects such as machines, vehicles and home appliances, are connected and accessed remotely by mobile devices connected to the Internet.

Therefore it is possible to affirm that the operation of a certain cyber physical system became conditioned along the operation of the Internet of Things.

\subsection{Internet of Services}

The Internet of Services (IoS) can be defined as a new way of business relation with its target audience through the intelligent objects, offering new services and transforming business models [14]. Through the IoS, both internal and external services are created, offered and reused by the value chain participants [12].

In a technical level, service can be a software available on Internet with a defined interface [15]; a platform used to pay for products through the web; or part of an infrastructure such as a virtual machine where files are stored, named "cloud" [16].

The services generated by the Internet of Services bring even more value to the Internet of Things because through the many objects connected, new services can be 
created and combined with each other. The cross-referencing of information through the companies and the users of the systems allows to add value to the service that is being provided to the final customer.

\section{$5 \quad$ Proposed architecture}

By the intersection of the technologies described, we propose an architecture for a Smart Contracts application on Blockchain technology as illustrated on Fig. 1.

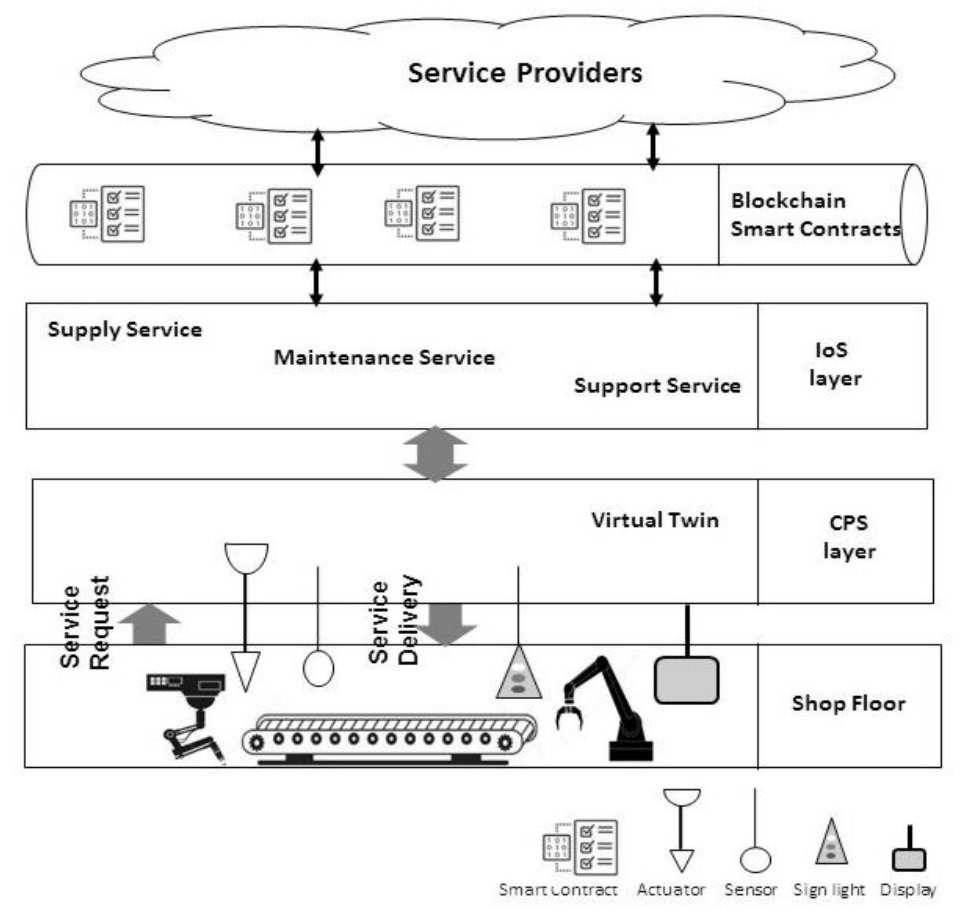

Fig. 1. Architecture for Blockchain and Smart Contracts through the Internet of Services

The proposed architecture works as follows:

- The Shop Floor (physical) layer is responsible for performing manufacturing transformation operations. In the case of productive processes, it can be understood as operation machines and tools, conveyor belts, robots, furnaces, boilers, manual operations, reactor tanks, etc. The events in this layer can request services (e.g. task validation; job information, poka yoke digitalization).

- The Cyber-Physical System (CPS) layer is interconnected to the physical elements through sensors and actuators, so that the monitoring and control of the physical environment can be performed from the virtual environment. In addition to the control of such equipment, it is also allowed to carry out tests and simulations. 
"Smart" equipment allow for decentralized decision-making and real-time human cooperation. The CPS layer delivers services to the Shop Floor layer through actuators, displays, sign lights, etc.

- The Internet of Services layer works as a service bus where different services can be accessed, matched and integrated by discovery and composition applications for an Industry 4.0 smart manufacturing environment [17].

- The Blockchain layer is implemented by Blockchain DLT (Distributed Ledger Technologies) allowed with an immutable ledger that will be maintained by peers in a private network where all Smart Contracts can be accessed. When a service is requested and delivered, a smart contract is stablished between the company and one service provider in a peer-to-peer transaction, validated by the Blockchain network. Thus, the Blockchain layer is responsible for:

- The validation of the predefined rules (services), in form of Smart Contracts, according to the distributed network consensus and stored in the specific Blockchain;

- The interactions on the development of new rules, as well as the adversity of operations, that come from the interconnection existing through the Internet of Services;

- The control, which is automatized and decentralized in the units, with their intelligent and autonomous objects on the production line.

- The Services Providers are different players, both virtual and physical, that, through the Blockchain validation, become trusted third parties within a collaborative business ecosystem where the services are offered and consumed in combined use.

The total integration of the value chain with the production structure allows possible communications with suppliers and customers through the Internet of Services.

\section{Application Case}

The proposed architecture can be illustrated considering the following scenario: A steel industry uses a complex emission filter system, provided by a partner through servitization.

Servitization in the manufacturing equipment industry is enabled by the increased incorporation of sensors that provides data about the condition and usage of manufacturing equipment. The collected data can be processed and analyzed to gain new insights and provides companies with the opportunity to create new value adding services [18].

For a product-oriented business model, the manufacturer has a low level of servitization. The revenue stream is largely based on product sales and spare parts and the ownership of the product is considered as transferred to the customer. This is a transaction-based "production and consumption" business model where the responsibilities of ownership lie with the customer. For a service-oriented business model, which has a higher level of servitization, the supplier still collocate the equipment, but the ownership of the product is not transferred to the customer. Instead, the supplier takes responsibility for equipment selection, consumables, monitoring of performance and 
carrying out servicing and disposal. In return, the supplier receives payment as the customer uses the capabilities that the equipment provides [19].

This service-oriented or "pay-per-use" model in a manufacturing company would rely on connected manufacturing equipment that contain embedded technologies, enabling them to interact with other objects or external environment.

\subsection{Context of the hypothetical case}

The steel industry "XYZ" presents a volatile productive capacity thus it demands elasticities, as well as pass-through rates and updating of allocation rules.

If an increase on production demand is forecasted, the filtering capacity should be flexible enough to match the new emission volume. On the other hand, when demand decreases to a lower level, the industry should not pay for a higher emission filtering.

To manage this critical configuration, software versioning and real-time access control are vital to maintain information quality and demand accuracy. Further, a connected network and "smart" equipment allow for decentralized and real-time decision-making.

Moreover, smart contracts may mitigate the bureaucracy and automate the contract rules by replacing the conventional service contracts.

In this "value in use" business model, the responsibilities for equipment performance lie with the supplier or service provider who receive revenues as the customer uses the filters.

\subsection{Application of proposed architecture}

By contracting the filtering capacity through Internet of Services and Blockchaintechnology, the contract rules between the steel industry "XYZ" and their service providers can be quickly adapted to the demand sensed by the filters on the shop floor level.

Illustrating through our proposed architecture, we have the following:

- Steel production filters on shop floor are on the physical layer with their sensors and actuators. Emission volume and emission composition sensors, that involves gases and particular material, requests services to the Cyber-Physical System (CPS) layer, depending on the up or down fluctuation.

- The Cyber-Physical System (CPS) layer is interconnected to such physical elements through their sensors and actuators, so that the monitoring and control of the physical environment can be performed from the virtual environment.

- Services can be invoked from the Internet of Services layer, such as Supplier services, Maintenance support service, among others. Regarding the emission volume control, the Supplier service is triggered for a contract update with the supplier.

- Depending on the gases composition, the contract model is one or another. A Smart Contract is managed via Blockchain, according to the filtering capacity of the virtual twin.

- The service provider, or supplier, validates the new level of gases emission and the Blockchain-based smart contract is updated on a peer-to-peer transaction. 
Such architecture addresses the self-adaptive contract, real-time flexitility and security, which are the challenges that the "XYZ" manufacturing company faces when developing its servitization model. It promotes a range of expected benefits that encourage manufactures to embrace the Blockchain technology.

\section{$7 \quad$ Final considerations}

This paper brings a conceptual Blockchain-technology architecture as a solution that can be implemented on manufacturing environment. It may validate certain manufacturing service demands from members of the Internet of Services context, through the rules and definitions of the Smart Contracts.

The services generated by the Internet of Services bring value to the Cyber Physical Systems in a manufacturing environement. By involving such Industry 4.0 structural elements and communication protocols, there is a great potential for creating rules or Smart Contracts through the Cyber Physical Systems, to be applied in validation and control of manufacturing processes. Through the data transmitted in real time, external elements can act in a productive system, controlling, monitoring and intervening both in a reactive or proactive way.

Nowadays, individuals and organizations are increasingly connected and using applications or services offered by companies through the crossings of information considered relevant to the final consumer. However in the current model, there is a need for a third party to validate a certain service. With applications in distributed systems and through the interaction and security offered by Blockchain technology, the validation will be defined by the network members themselves.

In a hypothetical case, we have presented an approach for implementing an adaptive servitization contract, based on the Blockchain proposed architecture.

\section{References}

1. Kagermann, H., Wahlster, W., Helbig, J.: Securing the future of German manufacturing industry: Recommendations for implementing the strategic initiative Industrie 4.0. Acatech - National Academy of Science and Engineering, pp. 1-82 (2013).

2. Sanders, A., Elangeswaren, C., Wulfsberg, J.: Industry 4.0 implies lean manufacturing: Research activities in industry 4.0 function as enablers for lean manufacturing, Journal of Industrial Engineering and Management, v. 9, n. 3, p. 811 (2016).

3. Hofmann, E., Rüsch, M.: Industry 4.0 and the current status as well as future prospects on logistics. Computers in Industry, v. 89, p. 23-34 (2017).

4. Witkowski, K.: Internet of things, big data, industry 4.0-innovative solutions in logistics and supply chains management. Procedia Engineering, v. 182, p. 763-769 (2017).

5. Autili, M., Giannakopoulou, D., Tivoli, M.: Thematic series on Verification and Composition for the Internet of Services and Things, Journal of Internet Services and Applications, 9 (1), art. no. 10 (2018).

6. Li, Z., Wang, W. M., Liu, G., Liu, L., He, J., Huang, G. Q.: Toward open manufacturing: A cross-enterprises knowledge and services exchange framework based on blockchain and edge computing. Industrial Management \& Data Systems, 118(1), 303-320 (2018). 
7. Tapscott, D., Tapscott, A.: Blockchain revolution: how the technology behind bitcoin is changing money, business, and the world. Penguin (2016).

8. Kweon, M.: A Study of Blockchain Technology in Facilitating Lending Services with Distributed Risk Aversion. Review of Computational Science and Engineering, v. 4, n. 1, p. 91 (2018).

9. Bitfury Group, Smart contracts on Bitcoin blockchain, <http://bitfury.com/content/5white-papers-research/contracts-1.1.1.pdf, last accessed 2019/02/18.

10. Gill, A. Q., Braytee, A., Hussain, F. K.: Adaptive Service E-Contract Information Management Reference Architecture. VINE Journal of Information and Knowledge Management Systems 47 (3): 395-410 (2017).

11. Givehchi, O., Landsdorf, K., Simoens, P., Colombo, A. W.: Interoperability for industrial Cyber Physical systems: an approach for legacy systems, in IEEE Transactions on Industrial Informatics, vol. PP, no. 99, pp. 1-1 (2017).

12. Hermann, M., Pentek, T., Otto, B.: Design principles for industrie 4.0 scenarios: a literature review. In: Working Paper No. 01/ 2015, Technische Universität Dortmund, Fakultät Maschinenbau and Audi Stiftungslehrstuhl - Supply Net, Order Management (2015).

13. Liu, Q., An Application of Horizontal and Vertical Integration in Cyber Physical Production Systems, International Conference on Cyber-Enabled Distributed Computing and Knowledge Discovery, Xi'an, pp. 110-113 (2015).

14. Satyro, W. C., Sacomano, J. B., da Silva, M. T., Gonçalves, R. F., Contador, J. C., Von Cieminski, G.: Industry 4.0: Evolution of the Research at the APMS Conference. In: IFIP International Conference on APMS 2017, p 39-47 (2017).

15. Soriano, J., Heitz, C., Hutter, H., Fernández, R.: Internet of Services. In: Evolution of Telecommunication Services. In: Springer Berlin Heidelberg. p 283-323 (2013).

16. Vozmediano, R. M., Montero, R. S., Llorente, I. M.: Key Challenges in Cloud Computing: Enabling the Future Internet of Services - IEEE Journals \& Magazine (2011).

17. Zonichenn Reis, J., Gonçalves, R., 2018. The Role of Internet of Services (IoS) on Industry 4.0 Through the Service Oriented Architecture (SOA): IFIP WG 5.7 International Conference, APMS 2018, Seoul, Korea, August 26-30, 2018, Proceedings, Part II. pp. 20-26. https://doi.org/10.1007/978-3-319-99707-0_3

18. European Commission, Cross-Cutting Business Models for IoT, European Union: European Commission (2017).

19. Samuelsson, S. Evaluating Servitization in the Manufacturing Equipment Industry (2018). 